\title{
Sensitive Detection of Silver Ions Based on Chiroplasmonic Assemblies of Nanoparticles
}

\author{
Zhou Xu, Liguang Xu, Luis M. Liz-Marzán, Wei Ma, Nicholas A. Kotov, Libing Wang, \\ Hua Kuang,* and Chuanlai Xu*
}

Chirality in nanocolloids originates from the absence of a plane of symmetry in complex nanoparticles (NPs) and their assemblies. ${ }^{[1-7]}$ In a broader sense it can be considered to be a consequence of dimensional and chemical similarity between NPs and globular biomacromolecules ${ }^{[8]}$ which also manifests in their optical activity with respect to circularly polarized (CP) photons when different extinction values are recorded when illuminated using left and right CP light. ${ }^{[9-11]}$ Chirality is particularly strong for plasmonic nanoparticles ${ }^{[12,13]}$ and plasmonic chiral nanomaterials ${ }^{[14,15]}$ have found applications in the sensitive characterization of biomolecules. ${ }^{[16]}$ The spatial arrangement, ${ }^{[17-19]}$ morphology, ${ }^{[20-22]}$ gap distance, ${ }^{[23-25]}$ and plasmonic coupling intensity ${ }^{[26]}$ of nanoparticles (NPs) are key factors in determining plasmonic chiroptical response. Helical assemblies of plasmonic NPs ${ }^{[27]}$ and other $\mathrm{NPS}^{[28]}$ can further enhance the optical activity. Despite some important advances in plasmonic chirality, the systematic understanding and utilization of this property are still considered an elusive pursuit.

Early work by our team demonstrated the fabrication of plasmonic chiral assemblies with intense chiroptical effects. ${ }^{[29]}$ Soon thereafter, 2D origami, ${ }^{[30,31]}$ supramolecular fibers, ${ }^{[32]}$ antibody-antigen reaction ${ }^{[33]}$ and DNA scaffolds, ${ }^{[29]}$ were used to fabricate plasmonic chiral architectures with different geometries, such as ladder superstructures, ${ }^{[34]}$ helices ${ }^{[30]}$ and pyramids, ${ }^{[35]}$ among others. As these studies usually involve complex assemblies with specific multi-NPs spatial arrangement, a main obstacle remains the poor understanding of the nature

Dr. Z. Xu, Dr. L. Xu, Dr. W. Ma, Prof. L. Wang, Dr. H. Kuang, Prof. C. Xu

State Key Lab of Food Science \& Technology

School of Food Science \& Technology

Jiangnan University

Wuxi, 214122, China

E-mail: khecho@163.com; xcl@jiangnan.edu.cn

Dr. Z. Xu

School of Chemistry and Biology Engineering

Changsha University of Science \& Technology

Changsha, 410114, China

Prof. L. M. Liz-Marzán

CIC biomaGUNE, Paseo de Miramón 182

San Sebastian, 20009, Spain

Prof. L. M. Liz-Marzán

Ikerbasque, Basque Foundation for Science

Bilbao, 48011, Spain

Prof. N. A. Kotov

Department of Chemical Engineering

University of Michigan

Ann Arbor, Michigan, 48109, USA

DOI: 10.1002/adom.201300148 of plasmonic chirality in each case. Simutaneously, the limited recognition ability of biomolecules (e.g. antibodies) toward various analytes and loss of their recognition activity upon binding on nanomaterials (e.g. the recognition site of antibodies may bind on the surface of nanomaterials) make these biomolecules unsuitable for the design of high stability sensors. Thus, developing assemblies with a simple structure and intense chiroptical signal for applications in biosensing requires a careful design in which the nature of plasmonic chirality can be systematically evaluated. Herein, we report the preparation of discrete chiral assemblies that do not depend on a template and can feature the required characteristics. These assemblies show no tendency to aggregate, which is essential for repeatability in chiroptical activity due to low sedimentation coefficients. ${ }^{[36,37]}$ These assemblies do not require using a chiral template or an effective three-dimensional arrangement of the NPs, and therefore the simple assembly process can be easily and effectively controlled. We demonstrate that the formation of such chiroptical assemblies is selectively triggered by the presence of $\mathrm{Ag}^{+}$ ions, thus rendering the system an efficient and sensitive silver ion sensor.

Ionic silver species are considered hazardous in natural water environment due to their bioaccumulation and toxicity, and for this reason silver ions were chosen as a model system fordetection. The detection mechanism that we present here is based on changes of the circular dichroism (CD) spectrum when the assembly of plasmonic NP heterodimers takes place through metal-mediated base pair (cytosine- $\mathrm{Ag}^{+}-$cytosine) formation. As illustrated in Figure 1, we prepared gold nanoparticles (Au NPs) with two different sizes, 10 and $25 \mathrm{~nm}$, coated with terminal thiol-functionalized DNA3 or DNA4 (26 base-pairs, see Table S1), respectively. To enhance the colloidal stability of the Au NPs an electrostatic stabilizer was employed, dipotassium bis ( $p$-sulfonatophenyl) phenylphosphane dihydrate. The DNA3-10 nm Au NPs and DNA4-25 nm Au NPs were purified by repeated centrifugation and redispersion in 0.5x Tris-borate buffer. When the two DNA-functionalized Au NP probes were mixed in a sample solution containing the target silver ions, a recognition process ${ }^{[38-40]}$ driven by $\mathrm{Ag}^{+}$ promoted the assembly of the two types of $\mathrm{Au} \mathrm{NP}$ probes into heterodimers. This assembly markedly increased the bulk chiroptical activity of the solution, which could be readily detected by CD spectroscopy. Indeed, the increase of the CD intensity of the solution could be quantitatively correlated with the silver ion concentration, since a higher silver ion concentration leads to more extensive assembly of Au NPs to form heterodimers, with a concomitant enhancement of the CD intensity in the visible wavelength range. 


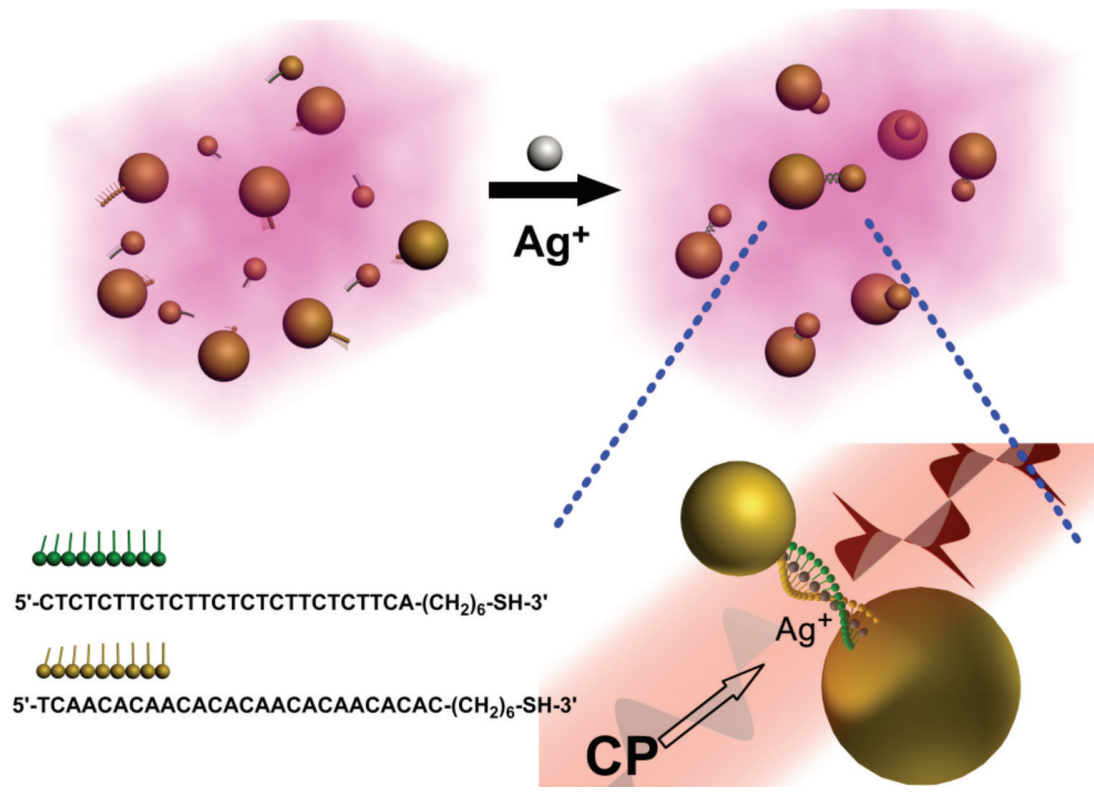

Figure 1. Schematic representation of the detection of $\mathrm{Ag}^{+}$ions based on Cyt-Ag-Cyt recognition between $10 \mathrm{~nm}$ and $25 \mathrm{~nm}$ Au NPs, each modified with different thiol-terminated cytosine-rich DNA3 or DNA4. Circular dichroism is measured as the difference in absorbance of circularly polarized light as a function of wavelength.

The minimum silver ion concentration required to induce heterodimer formation was determined by monitoring the yield of dimers as studied by transmission electron microscopy (TEM) images. We found that a concentration of silver ions as low as $10 \mathrm{nM}$ was sufficient to induce the formation of a high abundance of heterodimers (Figure 2A). To rule out the possibility of non-specific adsorption, and other assembly processes, ${ }^{[41]}$ a mixture of $10 \mathrm{~nm}$ Au NPs-DNA3 and $25 \mathrm{~nm}$ $\mathrm{Au}$ NPs-DNA4 was characterized by TEM (Figure 2A, inset), revealing the complete absence of dimers. The chemical composition of the gap was assessed by X-ray energy dispersive spectroscopy (XEDS) analysis in conjunction with TEM. As shown in Figure S1, the EDS spectrum showed the presence of two major elements: $\mathrm{Au}(99.5 \%)$ and $\mathrm{Ag}(0.5 \%)$. The $\mathrm{Au}$ and Ag peaks are expected to result from the heterodimer and DNA duplexes, confirming the presence of silver ions in the gap of the dimer.

The formation of chiral plasmonic heterodimers in solution was validated by the experimental CD spectra. As shown in Figure 2B, the absolute values of $\mathrm{CD}$ intensities in the UV region increased in the order: ssDNA (1.11 mdeg) < DNA duplexes (-3.87 mdeg) < dimer assembled by 26 bp DNA (-29.64 mdeg). The CD intensity of ssDNA is weak due to its random-coil structure composed of dNTP (chiral molecules). ${ }^{[42]}$ In the Vis/NIR range, the ssDNA, DNA duplexes, and Au NP-ssDNA did not exhibit any CD response above the random noise. However, a CD signal could be observed at $525 \mathrm{~nm}$ for the heterodimer assembled by DNA duplexes, which we assign to the chiral coupling of the LSPR modes of the Au NPs within the heterodimers (Figure 2B). Since a dimer made of two perfect spheres is in principle not expected to display chiral response (it does have a mirror image), we hypothesize that the observed CD signal arises because of the deviations of the NPs from sphericity, as confirmed by TEM (Figure 2A). The synthesized Au NPs could be considered as elongated ellipsoids, for which elongation factors of 1.29 and 1.21 were statistically calculated, for $25 \mathrm{~nm}$ and $10 \mathrm{~nm} \mathrm{Au} \mathrm{NPs,} \mathrm{respectively.} \mathrm{The} \mathrm{strong}$ chirality of the heterodimers is believed to be the result of plasmon-induced chirality affected by interference of the chiralmolecular dipole with two isolated coupled plasmons in the heterodimers (Supporting Information). ${ }^{[43]}$ The evidence of chiral properties of NP dimers and larger systems based on "cross-finger" geometries [26] can also be seen in other systems reported before. ${ }^{[3,44]}$ The chiral preference of one enatiomer over the other can originate from the tpreferential wisting of DNA which rotates of one elongated NP in respect to the other in clockwise or counterclockwise direction corresponding to the two possible chiral isomers. In this perspective, the NP dimers become similar to DNA-connected chiral nanorod dimers described in our recent work. ${ }^{[45]}$ Simulations based on the dimensions determined by TEM provided calculated CD responses that are in agreement with the experimental results (Figure 2B,C). The close match of the calculated and experimental spectra support this hypothesis although does not necessarily exclude the possibility of potential explanations of the origin of chirality in this system. It is clear, however that the observation of strong chiroptic activity in nanoparticle dimers connected by DNA indicates our limitation of knowledge of this seemingly simple system and their deviation from the common model of ideal spheres.

To develop the plasmonic chiral sensing system, we designed assemblies mediated by chiral molecules (DNA duplexes) and achiral molecules (D,L-dithiothreitol, D,L-DTT, mesomeric). Indeed, Figure 2B shows that the heterodimers formed by both chiral and achiral molecules gave rise to strong CD signals in the visible range, namely between 450 and $600 \mathrm{~nm}$, with high yields $(73.8 \%$ and $71.2 \%$, respectively) (Figure S2). The intensity of the chirality from the dimer formed by DNA ( $-116.3 \mathrm{mdeg})$ was significantly higher than that recorded from the dimer formed by DTT (-39.1 mdeg).

The detection performance of our system was optimized by systematically varying the gap distances between the NPs at the heterodimers, which was accomplished by using DNA strands made of 13, 26, and 39 base-pairs (bps) (see Table S1). The different gap distance between the heterodimers was expected to affect both the non-parallel polarized chirality ${ }^{[25]}$ and the plasmon-induced chirality. ${ }^{[26]}$ The results show that the CD saturated intensities at $525 \mathrm{~nm}$ for heterodimers linked by 13,26 , and 39 bps were $-68.88,-113.08$, and -33.61 mdeg, respectively (Figure 3 ). For the two heterodimers assembled by $13 \mathrm{bp}$ and $26 \mathrm{bp}$ DNA, the CD intensity at $525 \mathrm{~nm}$ was stronger than that of the heterodimer assembled by $39 \mathrm{bp}$ DNA, which can be explained in terms of the coupling theory 

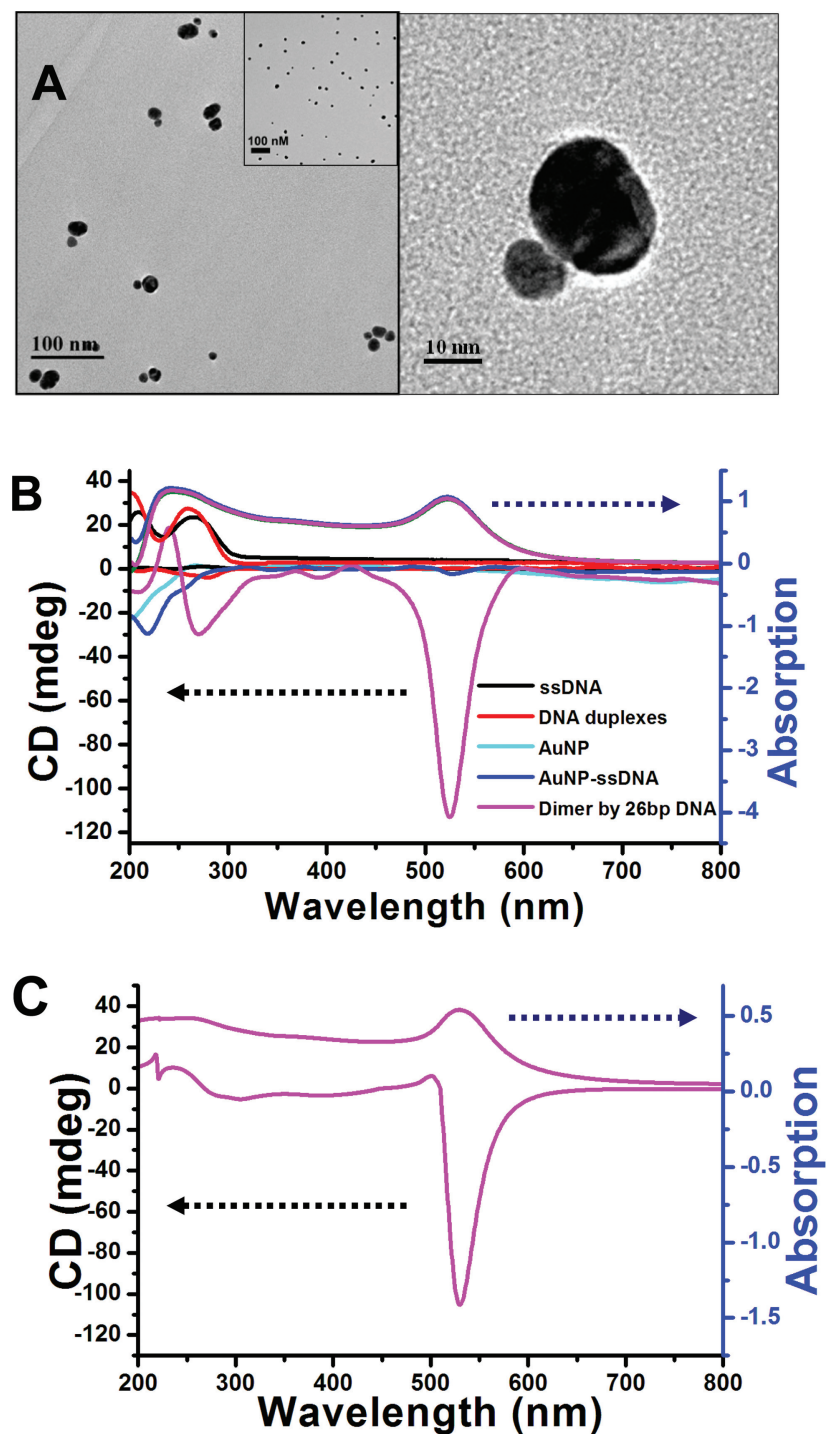

Figure 2. (A) Representative TEM image of heterodimers formed by silver ions (left). The inset shows a TEM image of a mixture of $10 \mathrm{~nm}$ Au NPs and $25 \mathrm{~nm}$ Au NPs prior to the addition of $\mathrm{Ag}^{+}$. Amplified TEM image of heterodimers formed by silver ions (right). (B) Overall CD spectra and UV/Vis absorbance spectra of ssDNA, DNA duplexes, Au NPs-ssDNA, dimer formed by DTT, and dimer formed by DNA. (C) Calculated CD spectra and UV/Vis absorbance spectra of dimer formed by silver ions DNA.

of non-parallel polarized chirality and the plasmon-induced chirality. The CD intensity from the heterodimer assembled by $26 \mathrm{bp}$ DNA was stronger than that from the heterodimer assembled by 13 bp DNA in solution. These differences could be related to (a) nanoscale mechanics of DNA bridges twisting the NPs in respect to each other or (b) lower yield of the heterodimer assembled by $13 \mathrm{bp}$ DNA as compared to that from the heterodimer assembled by 26 bp DNA (Figure 3B), and which may arise from steric hindrance of DNA hybridization and result in a lower likelihood of DNA interaction for the shorter DNA. For the heterodimer assembled by $26 \mathrm{bp}$
DNA, the detection sensitivity was higher than those assembled by 13 and 39 bp DNA, and the 26 bp DNA was selected to fabricate the model sensing system. Interestingly, the same trend has been obtained through calculations for the same Au NP dimensions and separation distances (see Supporting Information).

As shown in Figure 4A, when silver ions (our analyte) were added to the NP heterodimers (the mixture of DNA3-10 nm $\mathrm{Au}$ NPs and DNA4-25 $\mathrm{nm}$ Au NPs), the CD intensity at $525 \mathrm{~nm}$ increased significantly. The relationship between the CD intensity at $525 \mathrm{~nm}$ and the formation of heterodimers was statistically analyzed by measuring (using TEM) the yield of heterodimer formation at different $\mathrm{Ag}^{+}$concentrations (Figure 4C). We found that the yield of heterodimers indeed increased with increasing silver ion concentration. Furthermore, formation of the heterodimer had no obvious effect on the UV/vis absorbance spectrum, indicating that the $C D$ intensity was significantly more suitable as a detection signal and provided a much more sensitive detection performance. The anisotropy factor ( $g$-factor) can be used as a key indicator to evaluate the detection performance regardless of the detection system. The $\mathrm{g}$-factor of the plasmonic chiral assemblies can be as high as $1.6 \times 10^{-2}$ (Figure S6), which is significantly higher than those of liquid chromatography detection based on chiroptical activity $\left(9.9 \times 10^{-5}\right) \cdot{ }^{[46]}$ By plotting the variation in CD intensity at $525 \mathrm{~nm}$ at different concentrations of $\mathrm{Ag}^{+}$, a calibration curve was constructed as a function of logarithmic $\mathrm{Ag}^{+}$concentration (Figure 4B). This method showed a good correlation coefficient of $R^{2}=0.9913$, within a linear detection range of $0.005-10 \mathrm{nM}$, with a limit of detection (LOD) of $2 \mathrm{pM}$, which is lower than those typical of fluorescence assays, ${ }^{[38-40]}$ electrochemistry ${ }^{[47,48]}$ and atomic absorption spectrometry $(\mathrm{AAS})^{[49]}$ methods, which have often been regarded as the most sensitive assay methods. Moreover, the sensing parameters for silver ions surpass the required standard for drinking water by the US Environmental Protection Agency (EPA), which is $460 \mathrm{~nm}$. To examine the specificity of the detection system, we compared the detection of $\mathrm{Al}^{3+}, \mathrm{Zn}^{2+}, \mathrm{Mn}^{2+}, \mathrm{Hg}^{+}, \mathrm{Fe}^{2+}, \mathrm{Cu}^{2+}, \mathrm{Cr}^{6+}, \mathrm{Co}^{2+}$, and $\mathrm{Cd}^{2+}$. The $\mathrm{CD}$ intensities elicited by these ions $(100 \mathrm{nM})$ were weak, confirming that our system was highly specific for $\mathrm{Ag}^{+}$detection (Figure 4D).

In summary, we have successfully demonstrated a sensitive plasmonic chiroptical sensor based on Au NP heterodimers with good dispersibility, controllability, and repeatability. By self-assembly of Au NPs of two different sizes, mediated by both chiral and achiral molecules, we observed that plasmonic CD signals were obtained in the visible region. We believe that the chirality of the plasmonic heterodimers was a superposition of non-parallel polarized chirality (of slightly anisotropic NPs), while symmetry breaking between the two possible isomers-with clockwise and counterclockwise twistsoriginates from the mechanics of DNA bridges resulting in the twist preferentially in one direction. The distance was optimized, and under optimal conditions the method permitted the detection of $\mathrm{Ag}^{+}$ions with a LOD of $2 \mathrm{pM}$. This analytical method should find a variety of applications, including the chiral plasmon detection of small molecules, biomacromolecules, and DNA. 
Materials

www.MaterialsViews.com
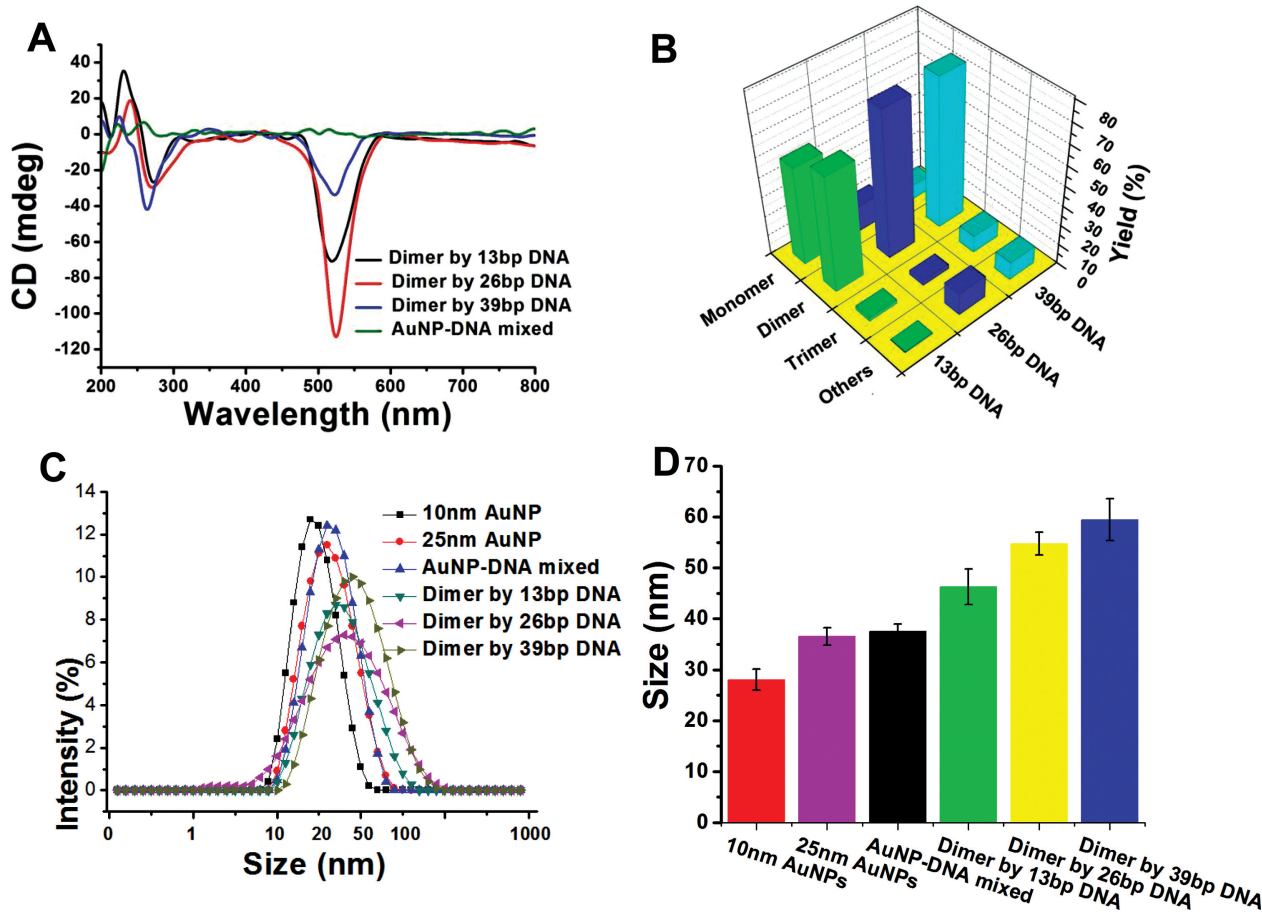

Figure 3. (A) DNA length-dependent CD spectra of chiral plasmon heterodimers. (B) Statistical analysis of the number of Au NPs assembled for different DNA lengths. Statistical analysis was carried out on the basis of different DNA lengths of assemblies classified as monomers, dimers, trimers, and others. (C) Dynamic light scattering size distributions of Au NPs in different states, as labeled. (D) Mean average hydrodynamic diameters of Au NPs in different states.
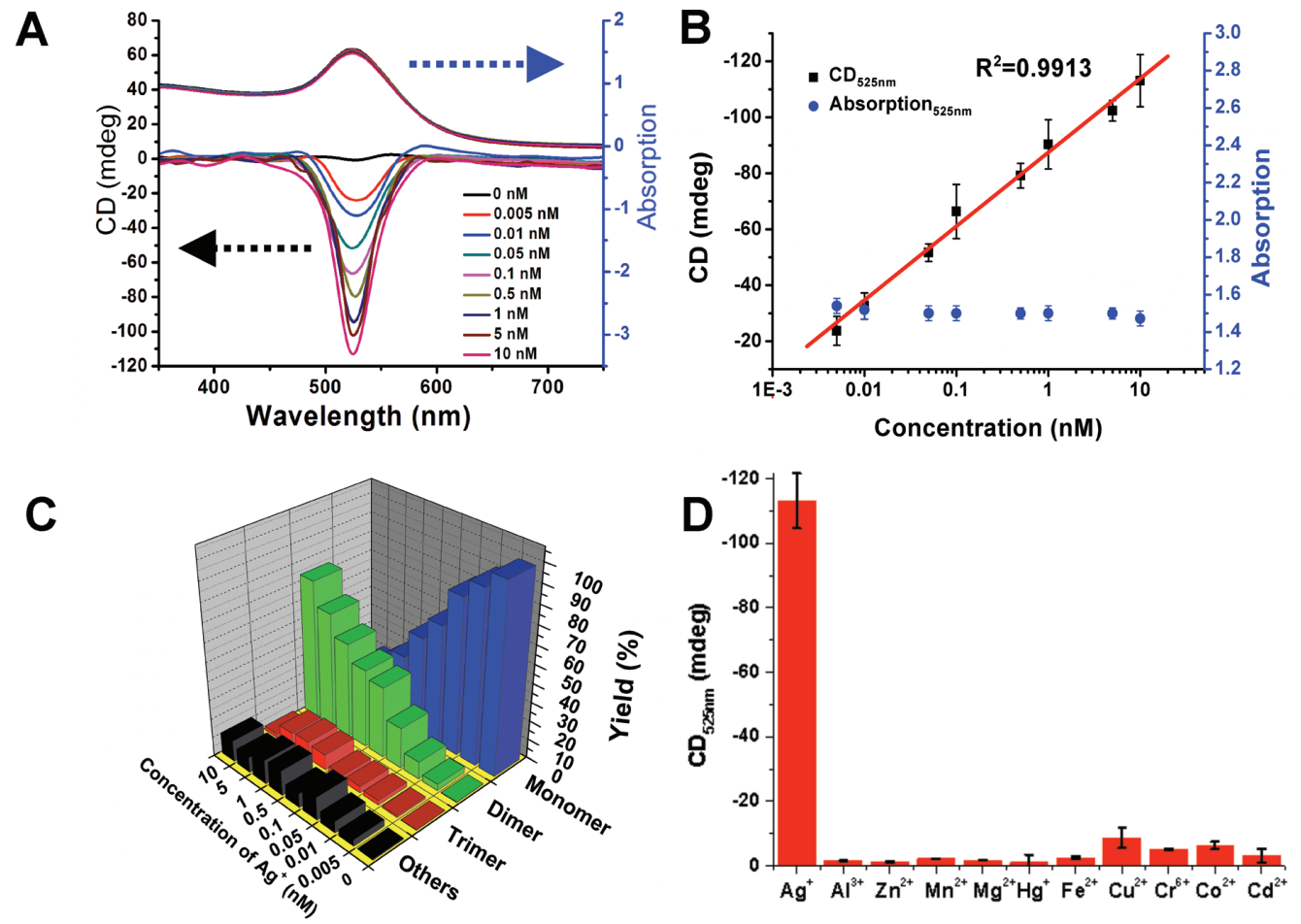

Figure 4. A) CD spectra and corresponding UV/Vis spectra after addition of different concentrations of silver ions. B) Plot of the variation in CD intensity at $525 \mathrm{~nm}$, as a function of logarithmic silver ion concentration, showing good linearity up to $10 \mathrm{nM}$, and no obvious change in absorption intensity at $525 \mathrm{~nm}$. C) Statistical analysis of the number of Au NPs assembled at different $\mathrm{Ag}^{+}$concentrations. Statistical analysis was carried out on the basis of different silver ion concentrations for assemblies classified as monomers, dimers, trimers, and others. D) Selectivity of the developed CD probe for silver ions over other metal ions. The concentration of each metal ion was $100 \mathrm{nM}$. 


\section{Supporting Information}

Supporting Information is available from the Wiley Online Library or from the author.

\section{Acknowledgements}

Z. $X u$ and L. $X u$ contributed equally to this work. This work is financially supported by the National Natural Science Foundation of China $(21071066,91027038,21101079,21175034)$, the Key Programs from MOST (2012BAC01B07, 2012AA06A303, 2012BAD29B04, 2012BAK11B01, 2011BAK10B07, 2011BAK10B01, 2010AA06Z302, 2010DFB3047, 2013ZX08012-001, 2012BAK17B10, 2012BAK08B01, 2012YQ090194), and grants from Jiangsu Province, MOF and MOE (NCET-12-0879, BE2011626, 201210036, 201310135, 311002).

Received: March 23, 2013 Revised: May 5, 2013 Published online: June 11, 2013

[1] Y. Zhou, M. Yang, K. Sun, Z. Tang, N. A. Kotov, J. Am. Chem. Soc. 2010, 132, 6006

[2] C. Helgert, E. Pshenay-Severin, M. Falkner, C. Menzel, C. Rockstuhl, E. B. Kley, A. Tünnermann, F. Lederer, T. Pertsch, Nano Lett. 2011, 11,4400

[3] J. E. Govan, E. Jan, A. Querejeta, N. A. Kotov, Y. K. Gun'ko, Chem. Commun. 2010, 46, 6072

[4] A. O. Govorov, Y. K. Gun'ko, J. M. Slocik, V. A. Gerard, Z. Y. Fan, R. R. Naik, J. Mater. Chem. 2011, 21, 16806.

[5] J. K. Gansel, M. Thiel, M. S. Rill, M. Decker, K. Bade, V. Saile, G. von Freymann, S. Linden, M. Wegener, Science 2009, 325, 1513.

[6] L. Wang, L. Xu, H. Kuang, C. Xu, N. A. Kotov, Acc. Chem. Res. 2012. 45, 1916.

[7] A. Guerrero-Martinez, J. L. Alonso-Gomez, B. Auguie, M. M. Cid, L. M. Liz-Marzan, Nano Today 2011, 6, 381.

[8] N. A. Kotov, Science 2010, 330, 188.

[9] M. Caricato, A. Olmo, C. Gargiulli, G. Gattuso, D. Pasini, Tetrahedron 2012, 68, 7861.

[10] M. Caricato, C. Coluccini, D. Dondi, D. A. Vander Griend, D. Pasini, Org. Biomol. Chem. 2010, 8, 3272.

[11] A. Moletti, C. Coluccini, D. Pasini, A. Taglietti, Dalton Trans. 2007, 16,1588

[12] T. G. Schaaff, R. L. Whetten, J. Phys. Chem. B 2000, 104, 2630.

[13] C. Gautier, T. Bürgi, J. Am. Chem. Soc. 2006, 128, 11079.

[14] J. Pendry, Science 2004, 306, 1353.

[15] A. Rogacheva, V. Fedotov, A. Schwanecke, N. Zheludev, Phys. Rev. Lett. 2006, 97, 177401.

[16] Z. T. Li, Z. N. Zhu, W. J. Liu, Y. L. Zhou, B. Han, Y. Gao, Z. Y. Tang, J. Am. Chem. Soc. 2012, 134, 3322.

[17] Z. Y. Fan, A. O. Govorov, J. Phys. Chem. C 2011, 115, 13254.

[18] M. Hentschel, M. Schaferling, T. Weiss, N. Liu, H. Giessen, Nano Lett. 2012, 12, 2542.

[19] Z. Zhu, W. Liu, Z. Li, B. Han, Y. Zhou, Y. Gao, Z. Tang, ACS Nano 2012, 6, 2326.

[20] Z. Y. Fan, A. O. Govorov, Nano Lett. 2012, 12, 3283.

[21] M. Ren, E. Plum, J. Xu, N. I. Zheludev, Nature Commun. 2012, 3, 833.
[22] K. Toyoda, K. Miyamoto, N. Aoki, R. Morita, T. Omatsu, Nano Lett. 2012, 12, 3645.

[23] S. V. Zhukovsky, C. Kremers, D. N. Chigrin, Optics Lett. 2011, 36, 2278.

[24] A. M. Funston, C. Novo, T. J. Davis, P. Mulvaney, Nano Lett. 2009, 9, 1651

[25] P. Nordlander, C. Oubre, E. Prodan, K. Li, M. Stockman, Nano Lett. 2004, 4, 899.

[26] N. A. Abdulrahman, Z. Fan, T. Tonooka, S. M. Kelly, N. Gadegaard, E. Hendry, A. O. Govorov, M. Kadodwala, Nano Lett. 2012, 12, 977.

[27] G. D. Lilly, A. Agarwal, S. Srivastava, N. A. Kotov, Small 2011, 7, 2004.

[28] S. Srivastava, A. Santos, K. Critchley, K.-S. Kim, P. Podsiadlo, K. Sun, J. Lee, C. Xu, G. D. Lilly, S. C. Glotzer, Science 2010, 327, 1355.

[29] W. Chen, A. Bian, A. Agarwal, L. Q. Liu, H. B. Shen, L. B. Wang, C. L. Xu, N. A. Kotov, Nano Lett. 2009, 9, 2153.

[30] X. Shen, C. Song, J. Wang, D. Shi, Z. Wang, N. Liu, B. Ding, J. Am. Chem. Soc. 2012. 134, 146.

[31] A. Kuzyk, R. Schreiber, Z. Y. Fan, G. Pardatscher, E. M. Roller, A. Hogele, F. C. Simmel, A. O. Govorov, T. Liedl, Nature 2012, 483, 311.

[32] A. Guerrero-Martínez, B. Auguié, J. L. Alonso-Gómez, Z. Džolić, S. Gómez-Graña, M. Žinić, M. M. Cid, L. M. Liz-Marzán, Angew. Chem. Int. Ed. 2011, 50, 5499.

[33] Z. Xu, L. Xu, Y. Zhu, W. Ma, H. Kuang, L. Wang, C. Xu, Chem. Commun. 2012. 48, 5760 .

[34] Y. Zhu, L. Xu, W. Ma, Z. Xu, H. Kuang, L. Wang, C. Xu, Chem. Commun. 2012, 48, 11889.

[35] W. Yan, L. Xu, C. Xu, W. Ma, H. Kuang, L. Wang, N. A. Kotov, J. Am. Chem. Soc. 2012, 134, 15114 .

[36] J. M. Zook, V. Rastogi, R. I. MacCuspie, A. M. Keene, J. Fagan, ACS Nano 2011, 5, 8070.

[37] L. Xu, H. Kuang, C. Xu, W. Ma, L. Wang, N. A. Kotov, J. Am. Chem. Soc. 2012, 134, 1699

[38] Y. Wen, F. Xing, S. He, S. Song, L. Wang, Y. Long, D. Li, C. Fan, Chem. Commun. 2010, 46, 2596.

[39] R. Freeman, T. Finder, I. Willner, Angew. Chem. Int. Ed. 2009, 48, 7818.

[40] A. Ono, S. Cao, H. Togashi, M. Tashiro, T. Fujimoto, T. Machinami, S. Oda, Y. Miyake, I. Okamoto, Y. Tanaka, Chem. Commun. 2008, 4825.

[41] L. Wang, L. Xu, H. Kuang, C. Xu, N. A. Kotov, Accounts of Chemical Research 2012, 45, 1916.

[42] A. Tomikawa, S. Kohgo, H. Ikezawa, N. Iwanami, K. Shudo, T. Kawaguchi, M. Saneyoshi, T. Yamaguchi, Biochem. Biophys. Res. Commun. 1997, 239, 329.

[43] B. Auguie, J. L. Alonso-Gomez, A. Guerrero-Martinez, L. M. Liz-Marzan, J. Phys. Chem. Lett. 2011, 2, 846.

[44] Y. Zhao, L. Xu, L. M. Liz-Marzán, H. Kuang, W. Ma, J. Garcia de Abajo, N. A. Kotov, L. Wang, C. Xu, J. Phys. Chem. Lett., 2013, 4, 641.

[45] W. Ma, H. Kuang, L. Wang, L. Xu, W. Chang, H. Zhang, M. Sun, Y. Zhu, Y. Zhao, L. Liu, C. Xu, S. Link, N. Kotov, Sci. Rep. 2013, DOI: 10.1038/srep01934.

[46] M. Lorin, R. Delépée, J. P. Ribet, P. Morin, J. Chromatography A 2007, 1141, 244

[47] C. Z. Lai, M. A. Fierke, R. C. Costa, J. A. Gladysz, A. Stein, P. Bühlmann, Analyt. Chem. 2010, 82, 7634.

[48] C. X. Tang, N. N. Bu, X. W. He, X. B. Yin, Chem. Commun. 2011, 47, 12304.

[49] P. Liang, L. Zhang, E. Zhao, Talanta 2010, 82, 993. 